Louisiana State University

LSU Digital Commons

$12-20-2006$

\title{
A search for neutrinos from the solar hep reaction and the diffuse supernova neutrino background with the Sudbury Neutrino Observatory
}

B. Aharmim

Laurentian University

S. N. Ahmed

Queen's University

A. E. Anthony

The University of Texas at Austin

E. W. Beier

University of Pennsylvania

A. Bellerive

Carleton University

See next page for additional authors

Follow this and additional works at: https://digitalcommons.Isu.edu/physics_astronomy_pubs

\section{Recommended Citation}

Aharmim, B., Ahmed, S., Anthony, A., Beier, E., Bellerive, A., Bergevin, M., Biller, S., Boulay, M., Chan, Y., Chen, M., Chen, X., Cleveland, B., Cox, G., Currat, C., Dai, X., Dalnoki-Veress, F., Deng, H., Detwiler, J., DiMarco, M., Doe, P., Doucas, G., Drouin, P., Duncan, F., Dunford, M., Dunmore, J., Earle, E., Evans, H., \& Ewan, G. (2006). A search for neutrinos from the solar hep reaction and the diffuse supernova neutrino background with the Sudbury Neutrino Observatory. Astrophysical Journal, 653 (2 I), 1545-1551.

https://doi.org/10.1086/508768

This Article is brought to you for free and open access by the Department of Physics \& Astronomy at LSU Digital Commons. It has been accepted for inclusion in Faculty Publications by an authorized administrator of LSU Digital Commons. For more information, please contact ir@lsu.edu. 


\section{Authors}

B. Aharmim, S. N. Ahmed, A. E. Anthony, E. W. Beier, A. Bellerive, M. Bergevin, S. D. Biller, M. G. Boulay, Y. D. Chan, M. Chen, X. Chen, B. T. Cleveland, G. A. Cox, C. A. Currat, X. Dai, F. Dalnoki-Veress, H. Deng, J.

Detwiler, M. DiMarco, P. J. Doe, G. Doucas, P. L. Drouin, F. A. Duncan, M. Dunford, J. A. Dunmore, E. D.

Earle, H. C. Evans, and G. T. Ewan 


\section{Sussex Research Online}

\section{A Search for Neutrinos from the Solar hep Reaction and the Diffuse Supernova Neutrino Background with the Sudbury Neutrino Observatory}

Article (Published Version)

Aharmim, B, Ahmed, SN, Anthony, AE, Peeters, S J M and et al, (2006) A Search for Neutrinos from the Solar hep Reaction and the Diffuse Supernova Neutrino Background with the Sudbury Neutrino Observatory. Astrophysical Journal (653). pp. 1545-1551. ISSN 0004-637X

This version is available from Sussex Research Online: http://sro.sussex.ac.uk/id/eprint/29693/

This document is made available in accordance with publisher policies and may differ from the published version or from the version of record. If you wish to cite this item you are advised to consult the publisher's version. Please see the URL above for details on accessing the published version.

\section{Copyright and reuse:}

Sussex Research Online is a digital repository of the research output of the University.

Copyright and all moral rights to the version of the paper presented here belong to the individual author(s) and/or other copyright owners. To the extent reasonable and practicable, the material made available in SRO has been checked for eligibility before being made available.

Copies of full text items generally can be reproduced, displayed or performed and given to third parties in any format or medium for personal research or study, educational, or not-for-profit purposes without prior permission or charge, provided that the authors, title and full bibliographic details are credited, a hyperlink and/or URL is given for the original metadata page and the content is not changed in any way. 


\title{
A SEARCH FOR NEUTRINOS FROM THE SOLAR hep REACTION AND THE DIFFUSE SUPERNOVA NEUTRINO BACKGROUND WITH THE SUDBURY NEUTRINO OBSERVATORY
}

B. Aharmim, ${ }^{1}$ S. N. Ahmed, ${ }^{2}$ A. E. Anthony ${ }^{3}$ E. W. Beier, ${ }^{4}$ A. Bellerive, ${ }^{5}$ M. Bergevin, ${ }^{6,7}$ S. D. Biller, ${ }^{8}$ M. G. Boulay, ${ }^{2,9}$ Y. D. Chan, ${ }^{6}$ M. Chen, ${ }^{2}$ X. Chen, ${ }^{6,10}$ B. T. Cleveland, ${ }^{8}$ G. A. Cox,${ }^{11}$ C. A. Currat,${ }^{6}$ X. Dai, ${ }^{2,8}$ F. Dalnoki-Veress ${ }^{5,12}$ H. Deng, ${ }^{4}$ J. Detwiler, ${ }^{11}$ M. DiMarco, ${ }^{2}$ P. J. Doe, ${ }^{11}$ G. Doucas, ${ }^{8}$ P.-L. Drouin, ${ }^{5}$ F. A. Duncan, ${ }^{2,13}$ M. Dunford, ${ }^{4}$ J. A. Dunmore, ${ }^{8,14}$ E. D. Earle, ${ }^{2}$ H. C. Evans, ${ }^{2}$ G. T. Ewan, ${ }^{2}$ J. Farine, $^{1}$ H. Fergani, ${ }^{8}$ F. Fleurot, ${ }^{1}$ R. J. Ford, ${ }^{2,13}$ J. A. Formaggio, ${ }^{11,15}$ N. Gagnon, ${ }^{6,8,9,11}$ J. Tm. Goon, ${ }^{16}$ K. Graham, ${ }^{2}$ E. Guillian, ${ }^{2}$ R. L. Hahn, ${ }^{17}$ A. L. Hallin, ${ }^{2}$ E. D. Hallman, ${ }^{1}$ P. J. Harvey, ${ }^{2}$ R. Hazama, ${ }^{11,18}$ K. M. Heeger, ${ }^{6,11}$ W. J. Heintzelman, ${ }^{4}$ J. Heise, ${ }^{13}$ R. L. Helmer, ${ }^{19,20}$ R. J. Hemingway, ${ }^{5}$ R. Henning, ${ }^{6}$ A. Hime, ${ }^{9}$ C. Howard, ${ }^{2}$ M. A. Howe, ${ }^{11}$ M. Huang, ${ }^{3}$ P. Jagam, ${ }^{7}$ N. A. Jelley, ${ }^{8}$ J. R. Klein, ${ }^{3,4}$ L. L. Kormos, ${ }^{2}$ M. Kos, ${ }^{2}$ A. Krüger, ${ }^{1}{ }^{\text {C. Kraus }, ~}{ }^{2}$ C. B. Krauss, ${ }^{2}$ T. Kutter, ${ }^{16}$ C. C. M. Kyba, ${ }^{4}$ H. Labranche, ${ }^{7}$ R. Lange, ${ }^{17}$ J. Law, ${ }^{7}$ I. T. Lawson, ${ }^{7,13}$ K. T. Lesko, ${ }^{6}$ J. R. Leslie, ${ }^{2}$ J. C. Loach, ${ }^{8}$ S. Luoma, ${ }^{1}$ R. Maclellan, ${ }^{2}$ S. Majerus, ${ }^{8}$ H. B. Mak, ${ }^{2}$ J. Maneira, ${ }^{21}$ A. D. Marino, ${ }^{6,22}$ R. Martin, ${ }^{2}$ N. McCauley, ${ }^{4,23}$ A. B. McDonald, ${ }^{2}$ S. McGee, ${ }^{11}$ C. Mifflin, ${ }^{5}$ K. K. S. Miknaitis, ${ }^{11,24}$ M. L. Miller, ${ }^{15}$ B. Monreal, ${ }^{15}$ B. G. Nickel, ${ }^{7}$ A. J. Noble, ${ }^{2}$ E. B. Norman, ${ }^{6,25}$ N. S. Oblath, ${ }^{11}$ C. E. Okada, ${ }^{6,26}$ H. M. O’ KeefFe, ${ }^{8}$ G. D. Orebi Gann, ${ }^{8}$ S. M. Oser,${ }^{20}$ R. Ott, ${ }^{15}$ S. J. M. Peeters, ${ }^{8}$ A. W. P. Poon, ${ }^{6}$ G. Prior,${ }_{1}^{6}$ K. Rielage, ${ }^{9,11}$ B. C. Robertson, ${ }^{2}$ R. G. H. Robertson, ${ }^{11}$ E. Rollin, ${ }^{5}$ M. H. Schwendener, ${ }^{1}$ J. A. Secrest, ${ }^{4}$ S. R. Seibert, ${ }^{3}$ O. Simard, ${ }^{5}$ C. J. Sims, ${ }^{8}$ D. Sinclair, ${ }^{5,19}$ P. Skensved, ${ }^{2}$ R. G. Stokstad, ${ }^{6}$ L. C. Stonehill, ${ }^{9,11}$ G. Teší,,${ }^{5}$ N. Tolich, ${ }^{6}$ T. Tsui, ${ }^{20}$ R. Van Berg, ${ }^{4}$ R. G. Van de Water, ${ }^{9}$ B. A. VanDevender, ${ }^{11}$ C. J. Virtue, ${ }^{1}$ T. J. Walker, ${ }^{15}$ B. L. Wall, ${ }^{11}$ D. Waller, ${ }^{5}$ H. Wan Chan Tseung, ${ }^{8}$ D. L. Wark, ${ }^{27,28}$ J. Wendland, ${ }^{20}$ N. West ${ }^{8}$ J. F. WILKerson, ${ }^{11}$ J. R. Willon, ${ }^{8,29}$ J. M. Wouters, ${ }^{9}$ A. Wright, ${ }^{2}$ M. YeH, ${ }^{17}$ F. ZHANG, ${ }^{5}$ AND K. ZuBER ${ }^{8,29}$ Received 2006 July 8; accepted 2006 August 28

\section{ABSTRACT}

A search has been made for neutrinos from the hep reaction in the Sun and from the diffuse supernova neutrino background (DSNB) using data collected during the first operational phase of the Sudbury Neutrino Observatory, with an exposure of 0.65 ktons yr. For the hep neutrino search, two events are observed in the effective electron energy range of $14.3 \mathrm{MeV}<T_{\text {eff }}<20 \mathrm{MeV}$, where 3.1 background events are expected. After accounting for neutrino oscillations, an upper limit of $2.3 \times 10^{4} \mathrm{~cm}^{-2} \mathrm{~s}^{-1}$ at the $90 \%$ confidence level is inferred on the integral total flux of hep neutrinos. For DSNB neutrinos, no events are observed in the effective electron energy range of $21 \mathrm{MeV}<$ $T_{\text {eff }}<35 \mathrm{MeV}$, and, consequently, an upper limit on the $\nu_{e}$ component of the DSNB flux in the neutrino energy range
\end{abstract}

\footnotetext{
1 Department of Physics and Astronomy, Laurentian University, Ramsey Lake Road, Sudbury, ON P3E 2C6, Canada.

2 Department of Physics, Queen's University, Kingston, ON K7L 3N6, Canada.

3 Department of Physics, University of Texas at Austin, Austin, TX 78712-0264.

4 Department of Physics and Astronomy, University of Pennsylvania, Philadelphia, PA 19104-6396.

5 Ottawa-Carleton Institute for Physics, Department of Physics, Carleton University, Ottawa, ON K1S 5B6, Canada.

6 Institute for Nuclear and Particle Astrophysics and Nuclear Science Division, Lawrence Berkeley National Laboratory, Berkeley, CA 94720.

7 Physics Department, University of Guelph, Guelph, ON N1G 2W1, Canada.

8 Department of Physics, University of Oxford, Denys Wilkinson Building, Keble Road, Oxford OX1 3RH, UK.

9 Los Alamos National Laboratory, Los Alamos, NM 87545.

${ }^{10}$ Current address: Goldman Sachs, 85 Broad Street, New York, NY 10004.

11 Center for Experimental Nuclear Physics and Astrophysics and Department of Physics, University of Washington, Seattle, WA 98195.

12 Current address: Physics Department, Princeton University, Princeton, NJ 08544-0708.

13 Sudbury Neutrino Observatory Laboratory, P.O. Box 146, Lively, ON P3Y 1M3, Canada.

14 Current address: Department of Physics, University of California, Irvine, CA 92697.

15 Laboratory for Nuclear Science, Massachusetts Institute of Technology, Cambridge, MA 02139.

16 Department of Physics and Astronomy, Louisiana State University, Baton Rouge, LA 70803.

17 Chemistry Department, Brookhaven National Laboratory, Upton, NY 11973-5000.

18 Current address: Graduate School of Engineering, Hiroshima University, Hiroshima 739-8526, Japan.

19 Tri-University Meson Facility, 4004 Wesbrook Mall, Vancouver, BC V6T 2A3, Canada.

20 Department of Physics and Astronomy, University of British Columbia, Vancouver, BC V6T 1Z1, Canada.

21 Laboratório de Instrumentação e Física Experimental de Partículas, 1000-149 Lisboa, Portugal.

22 Current address: Fermi National Accelerator Laboratory, Batavia, IL 60510.

23 Current address: Department of Physics, University of Liverpool, Liverpool L69 7ZE, UK.

24 Current address: Department of Physics, University of Chicago, Chicago, IL 60637.

25 Current address: Lawrence Livermore National Laboratory, Livermore, CA 94551.

26 Current address: Remote Sensing Laboratory, P.O. Box 98521, Las Vegas, NV 89193.

27 Rutherford Appleton Laboratory, Chilton, Didcot OX11 0QX, UK.

28 Also at: Imperial College, London SW7 2AZ, UK.

29 Current address: Department of Physics and Astronomy, University of Sussex, Brighton BN1 9QH, UK.
} 
of $22.9 \mathrm{MeV}<E_{\nu}<36.9 \mathrm{MeV}$ of $70 \mathrm{~cm}^{-2} \mathrm{~s}^{-1}$ is inferred at the $90 \%$ confidence level. This is an improvement by a factor of 6.5 on the previous best upper limit on the hep neutrino flux and by 2 orders of magnitude on the previous upper limit on the $\nu_{e}$ component of the DSNB flux.

Subject headings: neutrinos - Sun: general — supernovae: general

\section{INTRODUCTION}

The Sudbury Neutrino Observatory (SNO) is a real-time, heavy water Cerenkov detector located in the Inco, Ltd. Creighton nickel mine near Sudbury, Ontario, Canada at a depth of $6010 \mathrm{~m}$ water equivalent (Boger et al. 2000). SNO detects electrons and neutrons from, respectively, charged-current (CC) and neutral-current (NC) interactions of neutrinos on deuterons, as well as neutrinoelectron elastic scattering (ES) interactions, in $1 \mathrm{kton}$ of $\mathrm{D}_{2} \mathrm{O}$ contained in a $12 \mathrm{~m}$ diameter acrylic vessel (AV). These interactions are observed via Cerenkov light detected by 9456 photomultiplier tubes (PMTs) mounted on a $17.8 \mathrm{~m}$ diameter support structure. By comparing the observed rates of these interactions, SNO has demonstrated that a substantial fraction of the ${ }^{8} \mathrm{~B}$ electron neutrinos produced in the Sun transform into other active neutrino flavors (Ahmad et al. 2001, 2002a, 2002b, 2004; Aharmim et al. 2005). These results are consistent with the predictions of neutrino oscillations (Maki et al. 1962; Gribov \& Pontecorvo 1969; Wolfenstein 1978; Mikheyev \& Smirnov 1985).

The Sun generates energy by nuclear fusion; protons combine to form helium in reactions that release neutrinos. The primary solar fusion process is a series of reactions known as the $p p$ chain. Five reactions in the $p p$ chain produce neutrinos; the highest energy neutrinos are those from the hep reaction: ${ }^{3} \mathrm{He}+p \rightarrow{ }^{4} \mathrm{He}+$ $e^{+}+\nu_{e}$. The endpoint of the hep neutrino spectrum is $18.77 \mathrm{MeV}$ and lies above that of the ${ }^{8} \mathrm{~B}$ spectrum, which is approximately $15 \mathrm{MeV}$. The flux of hep neutrinos (e.g., Bahcall \& Krastev 1998; Bahcall \& Pinsonneault 2004) is currently predicted to be $(7.97 \pm$ $1.24) \times 10^{3} \mathrm{~cm}^{-2} \mathrm{~s}^{-1}$ (Bahcall et al. 2006), ${ }^{30}$ which is small compared to the fluxes from the other neutrino-producing reactions in the $p p$ chain, including the ${ }^{8} \mathrm{~B}$ flux, which has been measured to be $(4.95 \pm 0.42) \times 10^{6} \mathrm{~cm}^{-2} \mathrm{~s}^{-1}$ (Aharmim et al. 2005). The dominant contribution to the uncertainty in the hep neutrino flux prediction is $15.1 \%$ from the calculation of the nuclear matrix elements (Park et al. 2003). The previous best upper limit on the hep neutrino flux is $7.3 \times 10^{4} \mathrm{~cm}^{-2} \mathrm{~s}^{-1}$ at the $90 \%$ confidence level (CL), based on measurements with the Super-Kamiokande detector (Hosaka et al. 2006). After accounting for neutrino oscillations, this limit can be interpreted as an upper bound on the total flux of hep neutrinos of $1.5 \times 10^{5} \mathrm{~cm}^{-2} \mathrm{~s}^{-1}$. Currently, only one reaction $\left({ }^{8} \mathrm{~B}\right)$ from the $p p$ chain has been uniquely observed and measured experimentally. An observation of hep neutrinos would give further confirmation of the $p p$ chain as the primary solar energy generation mechanism and would allow further tests of the solar model.

Neutrinos produced in core-collapse supernovae also contribute to the energy region above the ${ }^{8} \mathrm{~B}$ endpoint. The current generation of neutrino detectors can detect the transient signal from a supernova in the Milky Way, but the expected signal from a supernova in a more distant galaxy is fewer than one event. Neutrinos from all extragalactic supernovae since the beginning of the formation of stars in the universe constitute the diffuse supernova neutrino background (DSNB), which may be detectable. Model predictions range from 0.19 to $1.49 \mathrm{~cm}^{-2} \mathrm{~s}^{-1}$ for the $\nu_{e}$

\footnotetext{
${ }^{30}$ The GS98 elemental abundances are selected for the reference model of solar neutrino fluxes.
}

component of the DSNB flux in the neutrino energy range $22.9 \mathrm{MeV}<E_{\nu}<36.9 \mathrm{MeV}$ (Beacom \& Strigari 2006; Ando $\&$ Sato $2003^{31}$ ). The best upper limit on the $\bar{\nu}_{e}$ component of the DSNB flux is $1.2 \mathrm{~cm}^{-2} \mathrm{~s}^{-1}$ at the $90 \% \mathrm{CL}$ for $E_{\bar{\nu}}>19.3 \mathrm{MeV}$, based on measurements with the Super-Kamiokande detector (Malek et al. 2003). While an indirect limit on the $\nu_{e}$ component of the DSNB flux can be inferred from this (Lunardini 2006), the previous best direct upper limit is $6.8 \times 10^{3} \mathrm{~cm}^{-2} \mathrm{~s}^{-1}$ for neutrino energies $25 \mathrm{MeV}<E_{\nu}<50 \mathrm{MeV}$, based on measurements with the Mont Blanc liquid scintillator detector (Aglietta et al. 1992).

A search for hep and DSNB neutrinos has been performed by counting the numbers of events in predefined energy intervals (signal boxes) above the ${ }^{8} \mathrm{~B}$ endpoint. The most sensitive signal boxes for this analysis were selected by evaluating the predicted signal and background levels before examining the data. Given the predicted signal and background levels in the signal boxes, limits on the flux of $h e p$ and DSNB neutrinos are set using a modified Feldman-Cousins technique. The following sections describe the data set, detector response, determination of the backgrounds, analysis procedures, and limits obtained for the hep and DSNB neutrino fluxes.

\section{THE DATA SET}

The data included in these analyses were collected during the initial phase of SNO operation, during which the detector contained pure $\mathrm{D}_{2} \mathrm{O}$. The data were collected from 1999 November 2 until 2001 May 28 and comprise 306.4 live days corresponding to an exposure of 0.65 ktons yr (Ahmad et al. 2002a).

Since results from this phase were last published, numerous improvements have been made to the analysis tools, many of which were used in the analysis of data from phase two (Aharmim et al. 2005), for which 2 tons of salt were dissolved in the heavy water. Further improvements were applied in this analysis, the most significant of which was improved estimation of the effective electron kinetic energies ( $\left.T_{\text {eff }}\right)$ of the events, based on the optical paths to each operational PMT. Other enhancements include improved handling of false hits due to cross talk between electronics channels and an improved accounting of working PMTs using both neutrino and calibration data to track bad channels. However, the vertex reconstruction algorithm was the same as that used in previous phase one analyses, in which events were reconstructed under the assumption that they are due to single electrons. This is more suited for the reconstruction of hep and DSNB events than the algorithm used in phase two. After the application of the new analysis tools, events inside the kinetic energy window of $12 \mathrm{MeV}<T_{\text {eff }}<35 \mathrm{MeV}$ were not examined until the hep and DSNB signal boxes had been selected.

In addition to the event selection discussed in Aharmim et al. (2005), which includes a selection that removes Michel electrons with visible precursors, selection criteria were applied to remove backgrounds from atmospheric neutrino interactions. As the hep and DSNB signals are expected to be single electron events, these backgrounds can be reduced significantly by removing events that

\footnotetext{
${ }^{31}$ Flux predictions from this paper have been increased by a factor of 3 , as recommended by the authors, to account for updated star formation rate data. Estimates for the $\nu_{e}$ DSNB fluxes were provided by the authors on request.
} 
correlate in time with neutrons, electrons, or $\gamma$-rays. Consequently, any candidate event that appeared within $250 \mathrm{~ms}$ of another with $T_{\text {eff }}>4 \mathrm{MeV}$ and a reconstructed vertex inside the AV was removed. In addition, two Kolmogorov-Smirnov (K-S) tests were applied: one to test the azimuthal symmetry of the PMT hits about the reconstructed event direction and the other to test the compatibility of the angular distribution of PMT hits with that expected from a single electron. In the signal boxes, the selections on PMT hit isotropy and the prompt light fraction were further tightened with respect to previous SNO analyses (Aharmim et al. 2005), which was possible in this analysis due to the higher energies of the candidate events. The combined event selection reduced the expected number of atmospheric neutrino events in the hep signal box by a factor of 29 and in the DSNB signal box by a factor of 77. The signal acceptance of the combined event selection is $96.6 \% \pm 0.7 \%$ for hep and $94.0 \% \pm 1.5 \%$ for DSNB events, measured using calibration source data and simulation.

\section{DETECTOR RESPONSE}

To understand the signals and backgrounds in this analysis, it is important to measure the energy response and uncertainties in the signal boxes. The energy response can be parameterized by a Gaussian of resolution $\sigma_{T}=-0.154+0.390 T_{e}^{1 / 2}+0.0336 T_{e}$, where $T_{e}$ is the true kinetic energy of the electron. In SNO analyses, Monte Carlo simulation is used to estimate the response of the detector to different particles. The propagation of electrons, positrons, and $\gamma$-rays is carried out using EGS4 (Nelson et al. 1985). The uncertainties in the energy scale and resolution of the SNO detector have typically been measured using $6.13 \mathrm{MeV}$ $\gamma$-rays from a ${ }^{16} \mathrm{~N}$ source (Dragowsky et al. 2002). At the higher energies more characteristic of this analysis, Michel electrons from muon decays and a $p T\left[{ }^{3} \mathrm{H}(p, \gamma){ }^{4} \mathrm{He}\right]$ source (Poon et al. 2000), which produces $19.8 \mathrm{MeV} \gamma$-rays, were used to complement the ${ }^{16} \mathrm{~N}$ measurements. Using simple event selection criteria, including one based on the time between events, 135 Michel electrons were identified in the data. Potential deviations in energy scale and energy resolution between data and simulations were assumed to be linear functions of energy. These functions were fit with a maximum likelihood technique using data from ${ }^{16} \mathrm{~N}$ and $p T$ sources as further constraints. The results were used to refine the energy scale and resolution estimates and to measure their uncertainties at the analysis thresholds. An energy scale uncertainty of $0.96 \%$ and a resolution uncertainty of $3.8 \%$ were estimated at the hep threshold of $14.3 \mathrm{MeV}$. At the DSNB threshold of $21 \mathrm{MeV}$, an energy scale uncertainty of $1.06 \%$ and a resolution uncertainty of $6.0 \%$ were estimated. Correlations between these quantities were included in the final analysis. Additional non-Gaussian tails to the resolution function were also considered but were found to be insignificant. Data and Monte Carlo distributions of $T_{\text {eff }}$ for ${ }^{16} \mathrm{~N}$ and $p T$ calibration events and for Michel electrons are shown in Figure 1.

Event vertex and direction reconstruction were unchanged from the analysis in Ahmad et al. (2002a). The position resolution at $15 \mathrm{MeV}$ is $12.0 \pm 2.5 \mathrm{~cm}$, and the angular resolution is $20^{\circ} .6 \pm 0.4$. These were measured using a combination of ${ }^{16} \mathrm{~N}$ source data and simulation. The same fiducial volume, defined by events reconstructed within a distance of $550 \mathrm{~cm}$ from the center of the detector, was selected. The uncertainty on the expected number of events within the fiducial volume due to vertex accuracy was $2.9 \%$.

\section{BACKGROUNDS}

Three distinct classes of background are considered: ${ }^{8} \mathrm{~B}$ neutrino interactions, atmospheric neutrino interactions, and instru-
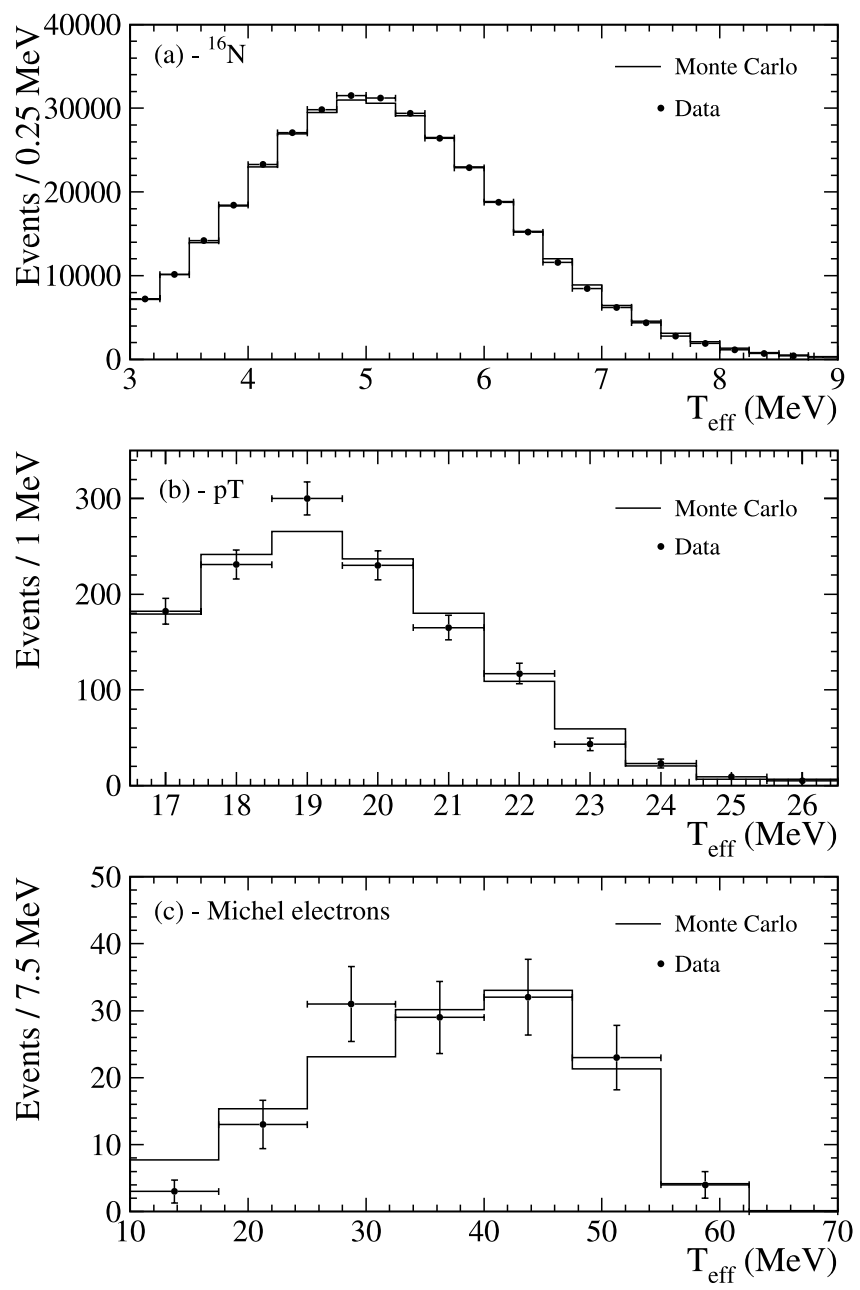

FIG. 1.-Effective electron kinetic energy spectra from data and Monte Carlo for $(a)$ events from the ${ }^{16} \mathrm{~N}$ source, $(b)$ events from the $p T$ source, and (c) Michel electrons. The data are shown in the energy regions free of source-related backgrounds.

mental backgrounds. Figure 2 shows the simulated energy spectra of the signals and backgrounds, normalized to their expected rates.

Electrons from ${ }^{8} \mathrm{~B}$ neutrino interactions are the dominant $(97 \%)$ background for the hep analysis but are a negligible background for DSNB. These events can reconstruct into the hep signal box due to the finite energy resolution of the detector. The magnitude of the ${ }^{8} \mathrm{~B}$ background depends on the details of the detector response and is very sensitive to the energy scale and resolution at threshold. In the CC interaction, by which SNO predominantly detects the ${ }^{8} \mathrm{~B}$ and hep neutrinos, there is a strong correlation between neutrino and electron energy. This, in addition to a cross section that rises with the square of the energy rather than linearly, provides a clearer distinction between the two neutrino spectra in the region of the ${ }^{8} \mathrm{~B}$ endpoint than is possible with the ES interaction.

The ${ }^{8} \mathrm{~B}$ background also depends on the details of the shape of the detected electron spectrum. The ${ }^{8} \mathrm{~B}$ neutrino spectrum from Winter et al. $(2003,2006)$ was assumed along with its quoted uncertainties. Neutrino oscillations were taken into account by correcting and combining the electron spectra from CC and ES interactions using the energy-dependent $\nu_{e}$ survival probability from the joint solar neutrino and KamLAND (Kamioka Liquid Scintillator Anti-Neutrino Detector; Araki et al. 2005) oscillation analysis presented in Aharmim et al. (2005). Additional spectral 


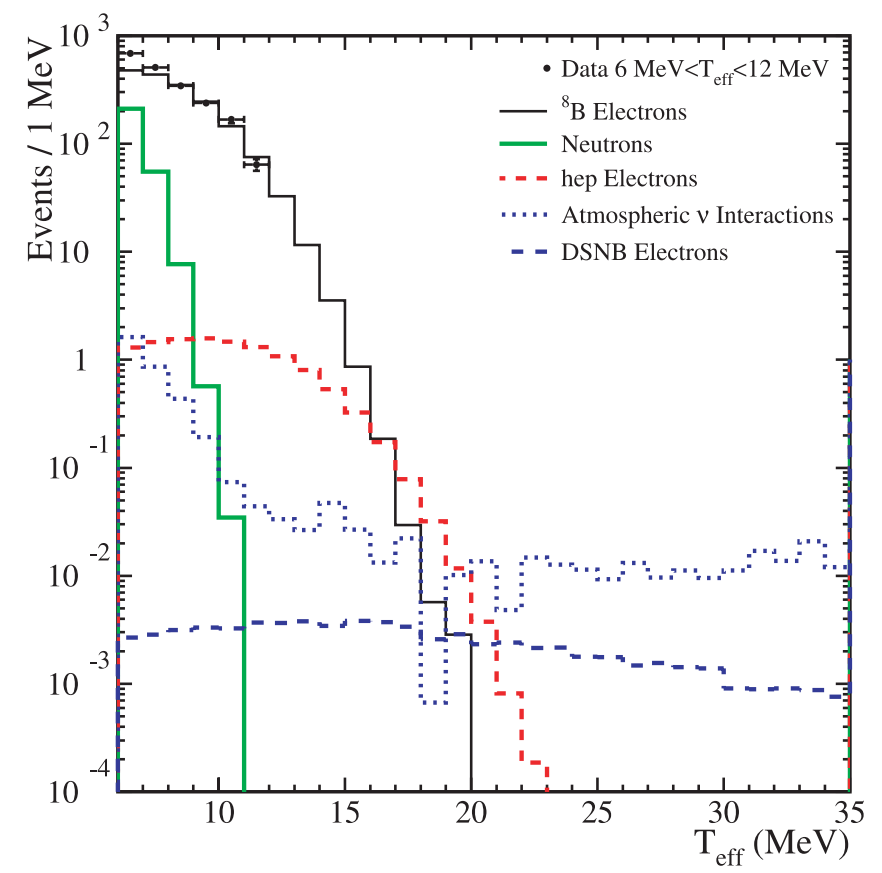

FIG. 2.- Simulated effective electron kinetic energy spectra of the signals and backgrounds of interest in the hep and DSNB analyses. Also shown are the data in the range $6 \mathrm{MeV}<T_{\text {eff }}<12 \mathrm{MeV}$ that are used to normalize the ${ }^{8} \mathrm{~B}$ electron and neutron distributions. The atmospheric neutrino background is made up of a number of different signals: neutrons at low energies, $\gamma$-rays from nuclear de-excitations at intermediate energies, and Michel electrons and CC interactions of atmospheric $\nu_{e}$ and $\bar{\nu}_{e}$ on deuterons at higher energies. The DSNB model in this figure is the $T=6 \mathrm{MeV}$ model from Beacom \& Strigari (2006). The third class of background, instrumental backgrounds, is negligible and is not shown in this figure.

adjustments were included to account for $\mathrm{CC}$ interactions on ${ }^{18} \mathrm{O}$, radiative corrections to the $\mathrm{CC}$ deuteron cross section (Nakamura et al. 2002; Kurylov et al. 2002), and the acceptance of the event selection. The expectation for the hep signal is constructed in the same way using the neutrino spectrum from Bahcall \& Ulrich (1988) with corrections from Bahcall (1997).

After the determination of the ${ }^{8} \mathrm{~B}$ signal shape, its normalization was determined using data at lower energies, where the hep signal is insignificant. In an energy window of $6 \mathrm{MeV}<T_{\text {eff }}<$ $12 \mathrm{MeV}, 2006$ events were observed. To determine normalizations, these data were fit using a maximum likelihood technique with probability density functions (PDFs) for the ${ }^{8} \mathrm{~B}$ electrons (CC and ES signals) and neutrons (NC signal and background). The distributions used in this fit were functions of event energy and direction with respect to the $\operatorname{Sun}\left(\cos \theta_{\odot}\right)$. The results of this fit were then used to estimate the ${ }^{8} \mathrm{~B}$ contribution inside the signal boxes.

Atmospheric neutrino interactions produce a second class of background events. They are the dominant background in the DSNB signal box and come from several sources:

1. Electrons from low-energy $\left(E_{\nu}<100 \mathrm{MeV}\right)$ charged-current $\nu_{e}$ and $\bar{\nu}_{e}$ interactions;

2. Michel electron events, in which the precursor muons (and pions) are below the Cerenkov threshold and do not trigger the detector;

3. $15.1 \mathrm{MeV} \gamma$-rays from de-excitation of an excited state of ${ }^{12} \mathrm{C}$ created via a nuclear cascade from neutrino interactions on ${ }^{16} \mathrm{O}$;

\section{Misidentified nonelectron events.}

For low-energy atmospheric $\nu_{e}$ and $\bar{\nu}_{e}$, the flux prediction from Battistoni et al. (2005) is used, which has an uncertainty of $25 \%$.
Only charged current interactions on deuterons, with cross sections from Nakamura et al. (2002) and Kurylov et al. (2002), are considered; the contributions from other interaction types are not significant. The interactions of these neutrinos constitute $14 \%$ of the DSNB background but are insignificant in the hep signal box.

Events from sources 2-4 are associated with atmospheric neutrinos of higher energy $\left(E_{\nu}>100 \mathrm{MeV}\right)$. Monte Carlo simulations were used to generate atmospheric neutrino interactions in the SNO detector with statistics equivalent to 500 times the expected number of events. For this purpose, the package NUANCE (Casper 2002) was used, ${ }^{32}$ with the Bartol04 flux prediction for Sudbury (Barr et al. 2004). The flux uncertainty in the neutrino energy range that contributes to the background is $10 \%$. The events generated by NUANCE were then propagated and fully simulated in the SNO Monte Carlo, from which background predictions were obtained after application of the event selection.

To assess uncertainties these events were divided into three categories. The first category, $\nu_{\mu}$ quasi-elastic (QE) CC events, is the primary source of untagged Michel electrons and originates from neutrinos in the energy range $150-250 \mathrm{MeV}$. The uncertainty on the cross section in this energy region is $25 \%$ (Barish et al. 1977). These Michel electrons comprise $80 \%$ of the DSNB background. For the second category, $15.1 \mathrm{MeV} \gamma$-ray events, there are no data in the literature on production rates, and thus a $100 \%$ uncertainty was assigned to the production rate predicted by NUANCE, which uses the calculation of Ejiri (1993). These $\gamma$-rays constitute half of the atmospheric background in the hep analysis, but due to the magnitude of the ${ }^{8} \mathrm{~B}$ background they constitute only $1.5 \%$ of the total hep background. The final category comprises QE NC events and interactions that produce pions, to which a cross section uncertainty of $30 \%$ is assigned (Ahrens et al. 1987).

There is an additional uncertainty applicable to the latter two categories of atmospheric neutrino interactions. A comparison of events from data and the simulation has shown that the simulation underestimates the production of correlated neutrons. It is unclear whether this is due to errors in the prediction of primary neutron production or in the transport of hadrons in the simulation. However, there is good agreement between data and Monte Carlo for correlated electron events. Events in the simulation are reweighted in such a way that the average neutron multiplicity is changed to better match the data. This results in a change to the background rejection rate in the simulation due to time-correlated neutrons. This correction results in an additional uncertainty of $7 \%$ in the rate of atmospheric background events inside the signal box that are not due to QE CC interactions. After application of the event selection to remove events with correlated neutrons, electrons, and $\gamma$-rays, the atmospheric background in these analyses is reduced by a factor of 2 .

To verify the predictions for the atmospheric neutrino background, data outside the signal box in the energy range $35 \mathrm{MeV}<$ $T_{\text {eff }}<55 \mathrm{MeV}$ were examined. This energy range was selected to be most sensitive to the main component of the atmospheric neutrino background: the Michel electrons. In this energy range, 0.28 Michel electrons and 0.05 electrons from low-energy chargedcurrent atmospheric neutrino interactions are expected. One event was observed, consistent with the predictions of the simulation. Inside this energy range, the effect of the event selection on events correlated with neutrons, electrons, or $\gamma$-rays was also examined. Two such events were observed, each consistent with being an otherwise untagged Michel electron preceded by a $\gamma$-ray from the de-excitation of the nucleus participating in the primary neutrino

32 NUANCE ver. 3 r009 was used in this analysis. 
TABLE 1

Major Uncertainties Included in the Analyses

\begin{tabular}{|c|c|}
\hline Source of Uncertainty & Magnitude of Effect \\
\hline \multicolumn{2}{|l|}{ Energy scale: } \\
\hline 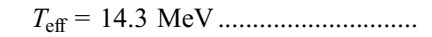 & $0.96 \%$ \\
\hline 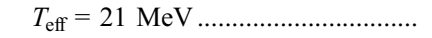 & $1.06 \%$ \\
\hline \multicolumn{2}{|l|}{ Energy resolution: } \\
\hline$T_{\mathrm{eff}}=14.3 \mathrm{MeV}$ & $3.8 \%$ \\
\hline$T_{\mathrm{eff}}=21 \mathrm{MeV}$ & $6.0 \%$ \\
\hline Vertex accuracy .............. & $2.9 \%$ \\
\hline Vertex resolution ........... & $2.5 \mathrm{~cm}$ \\
\hline Angular resolution ............................. & $2 \%$ \\
\hline${ }^{8} \mathrm{~B} \nu_{e}$ spectrum & a \\
\hline 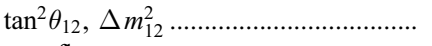 & $\mathrm{b}$ \\
\hline \multicolumn{2}{|l|}{$\nu_{\text {atm }}$ flux: } \\
\hline$E_{\nu}>100 \mathrm{MeV}$ & $10 \%$ \\
\hline$E_{\nu}<100 \mathrm{MeV}$ & $25 \%$ \\
\hline \multicolumn{2}{|l|}{ Cross sections: } \\
\hline $\mathrm{CC}$ deuteron ............. & $1.2 \%$ \\
\hline$\nu_{\text {atm }}(\mathrm{CC} \mathrm{QE}) \ldots \ldots \ldots . .$. & $25 \%$ \\
\hline 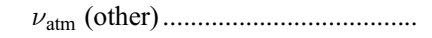 & $30 \%$ \\
\hline 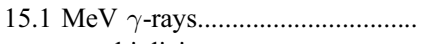 & $100 \%$ \\
\hline 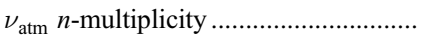 & $7 \%$ \\
\hline
\end{tabular}

Taken from Winter et al. (2006).

b Contours from Aharmim et al. (2005).

interaction. No events were observed that are correlated with neutron or electron events. These results are also consistent with the predictions of the simulation.

The final class of backgrounds is associated with instrumental effects such as electronic pickup or static discharge from the PMTs. For these events, an upper limit of 0.002 events is set in an energy range of $6 \mathrm{MeV}<T_{\text {eff }}<35 \mathrm{MeV}$ using the technique described in Aharmim et al. (2005). This analysis is not sensitive to the isotropic acrylic vessel background (IAVB; see Aharmim et al. 2005). To predict the number of IAVB events that pass the signal event selection, the 13 IAVB events clearly identified in the data by simple criteria are scaled via Monte Carlo simulation. A limit of $7 \times 10^{-4}$ IAVB events in the energy range of $14 \mathrm{MeV}<$ $T_{\text {eff }}<35 \mathrm{MeV}$ is inferred at the $90 \% \mathrm{CL}$.

\section{ANALYSIS AND RESULTS}

The analysis was designed to construct confidence intervals on the neutrino fluxes using a modified Feldman-Cousins approach (Feldman \& Cousins 1998; Conrad et al. 2003; Hill 2003). Limits were also calculated using a Bayesian approach (Eidelman et al. 2004); very similar results are obtained for the two techniques. To determine confidence limits the probability $p(N \mid S)$ of observing $N$ events, given a signal flux $S$, is calculated taking statistical fluctuations and all known systematic uncertainties into account. A Monte Carlo technique is used to integrate over the estimated distributions of the systematic uncertainties, including known correlations, by sampling ensembles of shifted parameter values and propagating their effect on the PDFs and extracted signal and background normalizations. The major uncertainties included in this procedure are shown in Table 1.

The hep and DSNB analyses are very similar, except that the definitions of signal and background are modified. In the DSNB analysis, the hep distribution is scaled using the standard solar model prediction, including its uncertainty, and added to the background estimate.

For the hep analysis the signal box was chosen to optimize the sensitivity based on Monte Carlo simulations. The sensitivity was
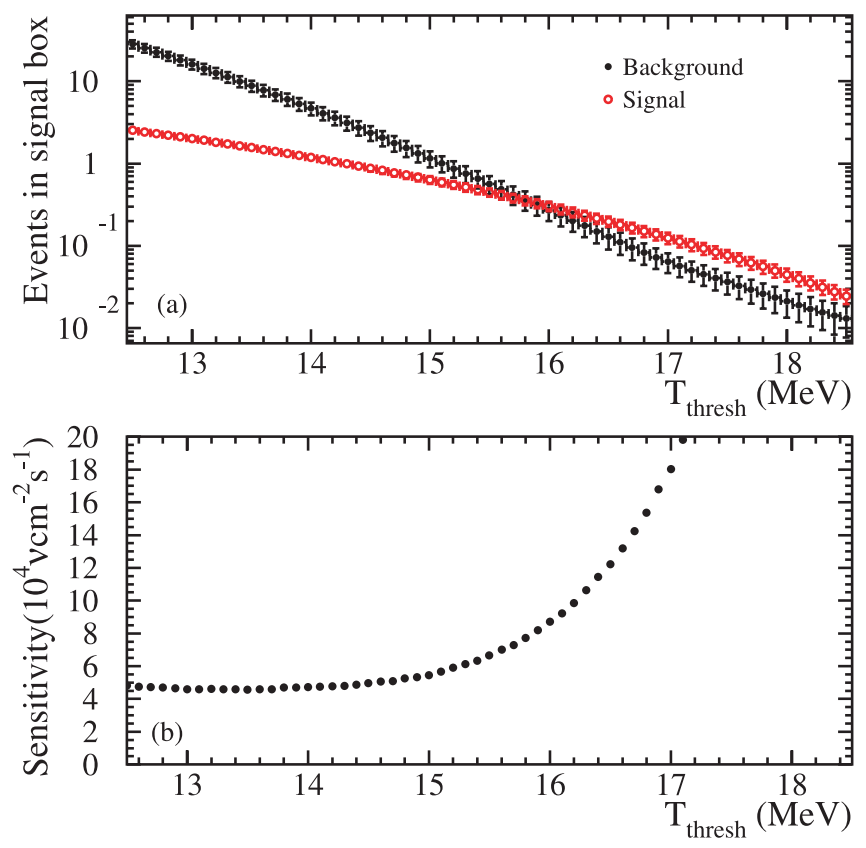

FIG. 3.- $(a, b)$ Expected number of events and the sensitivity of the analysis, respectively, as the energy threshold of the hep signal box is varied. The hep flux in $(a)$ is normalized to the solar model prediction. The upper limit of the signal box is fixed at $20 \mathrm{MeV}$.

defined as the mean value, from an ensemble of Monte Carlo experiments, of the $90 \%$ CL upper limit for the hep flux, integrated over all energies using the hep neutrino spectrum and accounting for neutrino oscillations as discussed in $\S 4$, assuming the standard solar model. Figure $3 a$ shows the predicted numbers of signal and background events with their $1 \sigma$ uncertainties as the lower threshold of the hep signal box is changed, and Figure $3 b$ shows the sensitivity of the analysis as a function of the signal box threshold. There is a region between 12.5 and $14.3 \mathrm{MeV}$ where the sensitivity is nearly flat. Within this range the choice of the best signal box is a compromise between the signal-to-background ratio and signal acceptance. The energy range $14.3 \mathrm{MeV}<T_{\text {eff }}<20 \mathrm{MeV}$ was selected. In this range, the variations of predicted signal and background levels due to systematic uncertainties are strongly correlated, as can be seen in Figure 4. In this signal box, $3.13 \pm 0.60$ background events and $0.99 \pm 0.09$ signal events are expected.

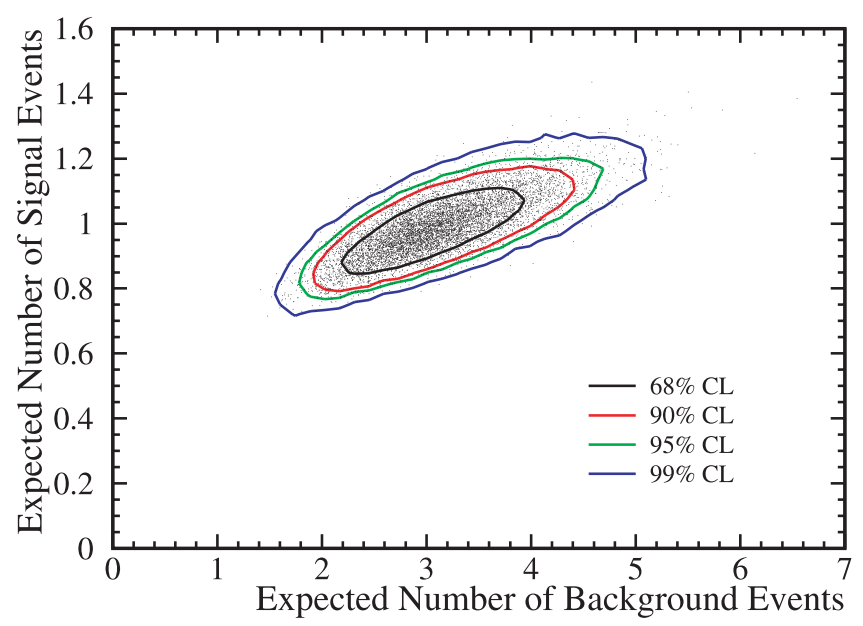

FIG. 4.- Joint probability distribution of signal and background events in the hep signal box assuming the standard solar model flux of hep neutrinos. 
TABLE 2

The Effect of Systematics on hep Signal and Background

\begin{tabular}{|c|c|c|}
\hline Systematic Source & $\begin{array}{c}\Delta \mathrm{Bkg} \\
(\%)\end{array}$ & $\begin{array}{c}\Delta \mathrm{Sig} \\
(\%)\end{array}$ \\
\hline Energy scale .......................... & 13.7 & 7.6 \\
\hline 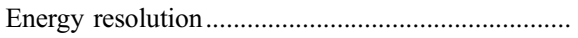 & 9.7 & 0.7 \\
\hline 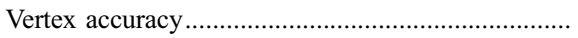 & 0.3 & 2.9 \\
\hline${ }^{8} \mathrm{~B} \nu_{e}$ spectrum $. . . \ldots .$. & 0.8 & 0.0 \\
\hline 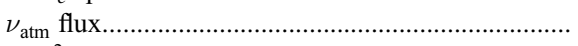 & 0.3 & 0.0 \\
\hline$\Delta m_{12}^{2} \ldots \ldots \ldots \ldots \ldots \ldots \ldots \ldots$ & 0.6 & 0.5 \\
\hline $\tan ^{2} \theta_{12} \ldots \ldots \ldots \ldots \ldots \ldots \ldots \ldots$ & 0.7 & 3.2 \\
\hline \multicolumn{3}{|l|}{ Cross sections: } \\
\hline $\mathrm{CC}$ deuteron .................... & 0.0 & 1.1 \\
\hline 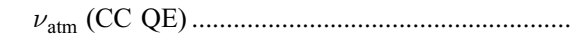 & 0.3 & 0.0 \\
\hline 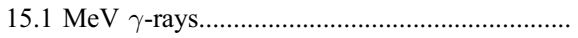 & 0.8 & 0.0 \\
\hline 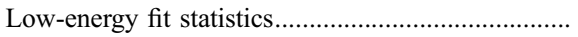 & 3.1 & 0.0 \\
\hline 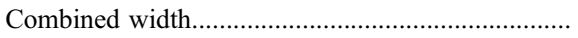 & 19.1 & 9.0 \\
\hline
\end{tabular}

Notes.-This table shows the $1 \sigma$ contributions to the width of the signal $(\Delta \mathrm{Sig})$ and background $(\Delta \mathrm{Bkg})$ probability distributions in the hep signal box. The combined widths are greater than the quadrature sums of the systematics due to correlations and nonlinearities.

The contributions to the signal and background uncertainties from the dominant sources of systematic uncertainties are shown in Table 2 .

Two events are observed in the hep signal box. After accounting for the effect of neutrino oscillations, this results in an upper limit on the integral total hep neutrino flux of $2.3 \times 10^{4} \mathrm{~cm}^{-2} \mathrm{~s}^{-1}$ at the $90 \%$ CL. This is 2.9 times the prediction of the standard solar model. Using a Bayesian technique rather than the modified Feldman-Cousins approach, a limit of $2.9 \times 10^{4} \mathrm{~cm}^{-2} \mathrm{~s}^{-1}$ is found at the $90 \% \mathrm{CL}$. The spectrum of events in the region of the signal box is shown in Figure 5. The shape agrees with the background prediction at the $77.8 \%$ CL based on Monte Carlo simulations using a K-S statistic.

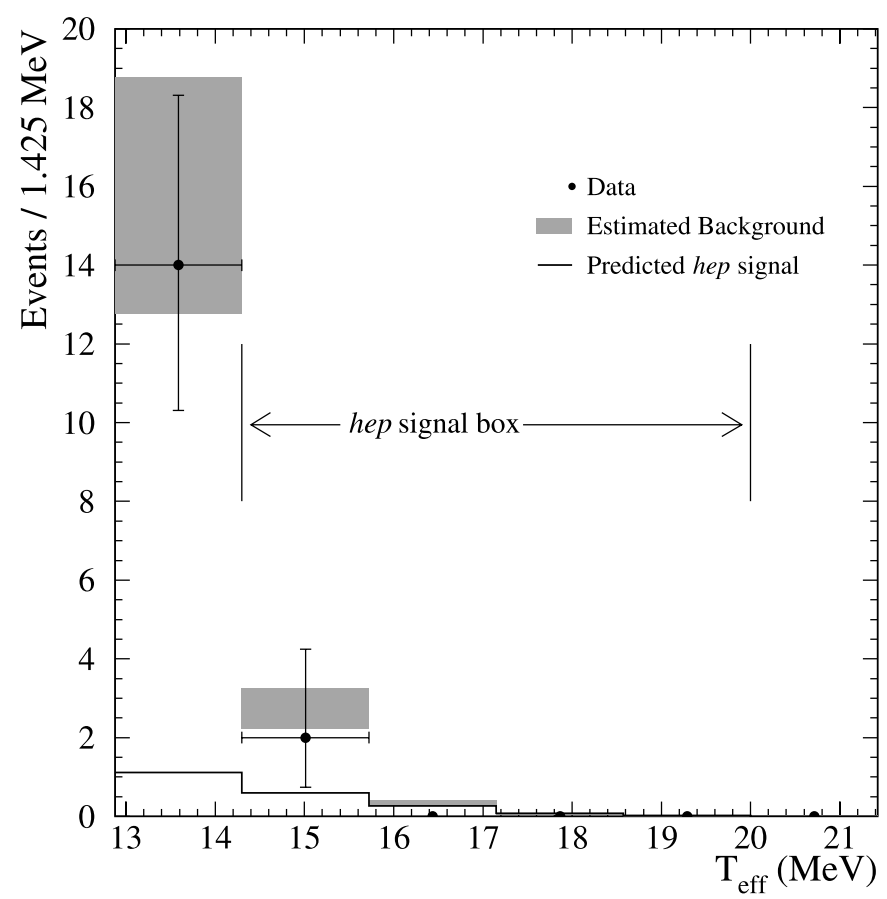

FIG. 5.-Distribution of events in the region of the ${ }^{8} \mathrm{~B}$ endpoint. There are two events in the hep signal box $14.3 \mathrm{MeV}<T_{\text {eff }}<20 \mathrm{MeV}$. Also shown is the estimated number of background events, including the systematic uncertainty, and the standard solar model prediction for the hep signal.
TABLE 3

The Effect of Systematics on DSNB Signal and Background

\begin{tabular}{|c|c|c|}
\hline Systematic Source & $\begin{array}{c}\Delta \mathrm{Bkg} \\
(\%)\end{array}$ & $\begin{array}{c}\Delta \mathrm{Sig} \\
(\%)\end{array}$ \\
\hline Energy scale........................ & 3.4 & 0.9 \\
\hline 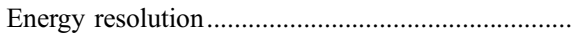 & 1.7 & 0.4 \\
\hline 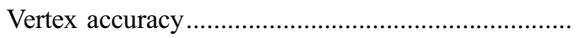 & 2.9 & 2.9 \\
\hline $\begin{array}{l}\text { Event selection } \\
\nu \text { fluxes: }\end{array}$ & 0.9 & 1.7 \\
\hline$\nu_{\mathrm{atm}} E_{\nu}>100 \mathrm{MeV} \ldots \ldots$. & 8.6 & 0.0 \\
\hline 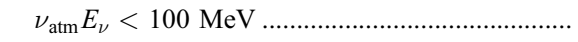 & 3.5 & 0.0 \\
\hline 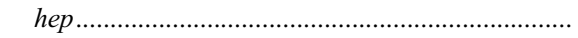 & 0.2 & 0.0 \\
\hline \multicolumn{3}{|l|}{ Cross sections: } \\
\hline 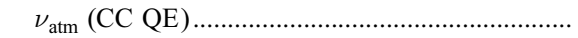 & 19.2 & 0.0 \\
\hline 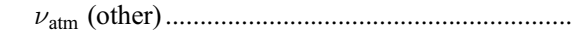 & 2.6 & 0.0 \\
\hline CC deuteron & 0.2 & 1.2 \\
\hline 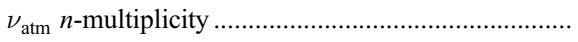 & 0.7 & 0.0 \\
\hline 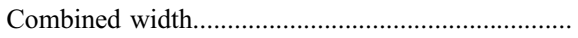 & 24.7 & 4.3 \\
\hline
\end{tabular}

Notes.-This table shows the $1 \sigma$ contributions to the width of the signal $(\Delta \mathrm{Sig})$ and background $(\Delta \mathrm{Bkg})$ distributions in the DSNB signal boxes. For the DSNB signal the $T=6 \mathrm{MeV}$ model from Beacom \& Strigari (2006) is used. As in the prediction for the signal and background in the hep signal box, combined widths are greater than the quadrature sums of the systematics.

This result is model-dependent, as neutrino oscillations were assumed in the predictions of signal and background. A modelindependent search, in which a limit is placed on the $\nu_{e}$ component of the integral hep flux, was also carried out. This search is independent of any model of neutrino flavor change. A further event selection of $\cos \theta_{\odot}<0.8$ was applied to remove ES events (which are directed away from the Sun) and leave events due only to CC $\left(\nu_{e}\right)$ interactions. The energy threshold was selected so that the mean background expectation was less than 0.25 events, and flux limits were set conservatively by assuming there is no background and quoting only the upper bound. With these criteria, a signal box of $16 \mathrm{MeV}<T_{\text {eff }}<20 \mathrm{MeV}$ was selected. Without neutrino oscillations $0.66 \pm 0.08$ hep events are expected in this box. As no events are observed a limit on the $\nu_{e}$ component of the integral hep neutrino flux of $3.1 \times 10^{4} \mathrm{~cm}^{-2} \mathrm{~s}^{-1}$ at the $90 \%$ $\mathrm{CL}$ is inferred.

Signal box selection for the DSNB search follows the approach of the hep neutrino search. A signal box of $21 \mathrm{MeV}<T_{\text {eff }}<$ $35 \mathrm{MeV}$ was selected in this case. This choice was bounded at the high end by the prior choice of the hidden energy interval and at the low end by a desire to minimize any contribution from hep neutrinos. In this signal box, $0.18 \pm 0.04$ background events are expected. The expected number of signal events depends on the assumed DSNB model but is uncorrelated with the background prediction as different systematic uncertainties are dominant for signal and background. The estimated values of the systematic uncertainties are shown in Table 3 . No events are observed in the signal box, resulting in an upper limit of 2.3 events due to DSNB neutrinos at the $90 \% \mathrm{CL}$.

To obtain a DSNB flux limit, a spectral model for the DSNB neutrinos is required. In this paper, three models from Beacom \& Strigari (2006) and two models from Ando \& Sato (2003) for differential flux predictions have been used. Table 4 shows the integral flux predictions for these models and the 90\% CL upper limits inferred from data.

Using these results, a limit can also be derived on the $\operatorname{DSNB} \nu_{e}$ flux for neutrinos that produce electrons with kinetic energies inside the DSNB signal box. Although the integral flux upper limits are significantly different for these models, since their spectral shapes are similar in the signal box, the resulting upper limits for 
TABLE 4

DSNB Flux Predictions and Limits

\begin{tabular}{|c|c|c|c|c|}
\hline \multirow[b]{2}{*}{ Model } & \multicolumn{2}{|c|}{$\begin{array}{l}\text { INTEGRAL FluX } \\
\left(\mathrm{cm}^{-2} \mathrm{~s}^{-1}\right)\end{array}$} & \multicolumn{2}{|c|}{$\begin{array}{c}\text { FLux } 22.9 \mathrm{MeV}<E_{\nu}<36.9 \mathrm{MeV} \\
\left(\mathrm{cm}^{-2} \mathrm{~s}^{-1}\right)\end{array}$} \\
\hline & Prediction & Upper Limit & Prediction & Upper Limit \\
\hline \multicolumn{5}{|c|}{ Beacom \& Strigari (2006) } \\
\hline 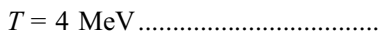 & 21.1 & $1.1 \times 10^{4}$ & 0.19 & 93 \\
\hline$T=6 \mathrm{MeV}$ & 14.1 & $1.5 \times 10^{3}$ & 0.66 & 72 \\
\hline$T=8 \mathrm{MeV}$ & 10.5 & $6.0 \times 10^{2}$ & 1.08 & 61 \\
\hline \multicolumn{5}{|c|}{ Ando \& Sato (2003) } \\
\hline NOR-L .................................... & 28.5 & $1.3 \times 10^{3}$ & 1.49 & 69 \\
\hline NOR-S-INV ............................... & 34.9 & $2.3 \times 10^{3}$ & 1.06 & 70 \\
\hline
\end{tabular}

NoтE.-This table shows the $90 \%$ CL upper limits on the $\nu_{e}$ component of the DSNB flux and model predictions for different models from Beacom \& Strigari (2006) and Ando \& Sato (2003).

the neutrinos in this region vary little (see Table 4). Taking the median result, a limit on the DSNB $\nu_{e}$ flux of $70 \mathrm{~cm}^{-2} \mathrm{~s}^{-1}$ at the $90 \% \mathrm{CL}$ for $22.9 \mathrm{MeV}<E_{\nu}<36.9 \mathrm{MeV}$ is inferred. These limits and the background prediction are in good agreement with those predicted by Beacom \& Strigari (2006) after accounting for the difference in exposure between their prediction and the data used in this search.

\section{CONCLUSIONS}

Data from the first operational phase of SNO, with an exposure of $0.65 \mathrm{ktons} \mathrm{yr}$, have been used to search for neutrinos from the hep reaction in the Sun. No evidence for these neutrinos was observed, and an upper limit on the integral total flux of hep neutrinos of $2.3 \times 10^{4} \mathrm{~cm}^{-2} \mathrm{~s}^{-1}$ has been derived at the $90 \% \mathrm{CL}$. This measurement improves the previous best limit on the hep neutrino flux, measured with the Super-Kamiokande detector, by a factor of 6.5 but is not inconsistent with the standard solar model. A model-independent limit on the integral $h e p \nu_{e}$ flux, with no assumptions about neutrino oscillations, is set at $3.1 \times 10^{4} \mathrm{~cm}^{-2} \mathrm{~s}^{-1}$. A search for the $\nu_{e}$ component of the diffuse supernova neutrino background has also been made using SNO data. Again, no evidence for these neutrinos was found, and an upper limit at $90 \%$
CL on the $\nu_{e}$ component of the DSNB flux of $70 \mathrm{~cm}^{-2} \mathrm{~s}^{-1}$ for $22.9 \mathrm{MeV}<E_{\nu}<36.9 \mathrm{MeV}$ is inferred. This is an improvement of 2 orders of magnitude on the previous $\nu_{e}$ limit (Aglietta et al. 1992). The exposure of the final SNO data set for these analyses combined across all phases of the experiment is expected to be approximately 4 times that of the data used in this analysis. A future search for hep and DSNB fluxes using these data will be carried out, which is expected to further improve on the limits presented in this paper.

This research was supported by the following institutions: Canada: Natural Sciences and Engineering Research Council, Industry Canada, National Research Council, Northern Ontario Heritage Fund, Atomic Energy of Canada, Ltd., Ontario Power Generation, High Performance Computing Virtual Laboratory, Canada Foundation for Innovation; US: Department of Energy, National Energy Research Scientific Computing Center, Alfred P. Sloan Foundation; UK: Particle Physics and Astronomy Research Council. We thank the SNO technical staff for their strong contributions. We thank Inco, Ltd. for hosting this project.
Aglietta, M., et al. 1992, Astropart. Phys., 1, 1

Aharmim, B., et al. 2005, Phys. Rev. C, 72, 055502

Ahmad, Q. R., et al. 2001, Phys. Rev. Lett., 87, 071301

. 2002a, Phys. Rev. Lett., 89, 011301

2002b, Phys. Rev. Lett., 89, 011302

Ahmed, S. N., et al. 2004, Phys. Rev. Lett., 92, 181301

Ahrens, L. A., et al. 1987, Phys. Rev. D, 35, 785

Ando, S., \& Sato, K. 2003, Phys. Lett. B, 559, 113

Araki, T., et al. 2005, Phys. Rev. Lett., 94, 081801

Bahcall, J. N. 1997, Phys. Rev. C, 56, 3391

Bahcall, J. N., \& Krastev, P. I. 1998, Phys. Lett. B, 436, 243

Bahcall, J. N., \& Pinsonneault, M. H. 2004, Phys. Rev. Lett., 92, 121301

Bahcall, J. N., Serenelli, A. M., \& Basu, S. 2006, ApJS, 165, 400

Bahcall, J. N., \& Ulrich, R. K. 1988, Rev. Mod. Phys., 60, 297

Barish, S. J., et al. 1977, Phys. Rev. D, 16, 3103

Barr, G. D., Gaisser, T. K., Lipari, P., Robbins, S., \& Stanev, T. 2004, Phys. Rev. D, 70, 023006

Battistoni, G., Ferrari, A., Montaruli, T., \& Sala, P. R. 2005, Astropart. Phys., 23,526

Beacom, J. F., \& Strigari, L. F. 2006, Phys. Rev. C, 73, 035807

Boger, J., et al. 2000, Nucl. Instrum. Methods Phys. Res. A, 449, 172

Casper, D. 2002, Nucl. Phys. B Proc. Suppl., 112, 161

Conrad, J., Botner, O., Hallgren, A., \& Pérez de los Heros, C. 2003, Phys. Rev. D, 67,012002

\section{REFERENCES}

Dragowsky, M. R., et al. 2002, Nucl. Instrum. Methods Phys. Res. A, 481, 284 Eidelman, S., et al. 2004, Phys. Lett. B, 592, 1

Ejiri, H. 1993, Phys. Rev. C, 48, 1442

Feldman, G. J., \& Cousins, R. D. 1998, Phys. Rev. D, 57, 3873

Gribov, V., \& Pontecorvo, B. 1969, Phys. Lett. B, 28, 493

Hill, G. C. 2003, Phys. Rev. D, 67, 118101

Hosaka, J., et al. 2006, Phys. Rev. D, 73, 112001

Kurylov, A., Ramsey-Muslof, M. J., \& Vogel, P. 2002, Phys. Rev. C, 65, 055501 Lunardini, C. 2006, Phys. Rev. D, 73, 083009

Maki, Z., Nakagawa, N., \& Sakata, S. 1962, Prog. Theor. Phys., 28, 870

Malek, M., et al. 2003, Phys. Rev. Lett., 90, 061101

Mikheyev, S. P., \& Smirnov, A. Yu. 1985, Soviet J. Nucl. Phys., 42, 913

Nakamura, S., Sato, T., Ando, S., Park, T.-S., Myhrer, F., Gudkov, V., \& Kubodera, K. 2002, Nucl. Phys. A, 707, 561

Nelson, W. O., Hirayama, H., \& Rogers, D. W. O. 1985, SLAC Rep. 265 (Menlo Park: SLAC)

Park, T.-S., et al. 2003, Phys. Rev. C, 67, 055206

Poon, A. W. P., Komar, R. J., Waltham, C. E., Browne, M. C., Robertson, R. G. H., Kherani, N. P., \& Mak, H. B. 2000, Nucl. Instrum. Methods Phys. Res. A, 452, 115

Winter, W. T., Freedman, S. J., Rehm, K. E., \& Schiffer, J. P. 2006, Phys. Rev. C, 73, 025503

Winter, W. T., et al. 2003, Phys. Rev. Lett., 91, 252501

Wolfenstein, L. 1978, Phys. Rev. D, 17, 2369 